\title{
Analytical Functions of Magnetization Curves for High Magnetic Permeability Materials
}

\author{
Mehran Mirzaei ${ }^{1}$, and Pavel Ripka ${ }^{1}$ \\ ${ }^{1}$ Faculty of Electrical Engineering, Czech Technical University, 16627 Prague, Czech Republic
}

\begin{abstract}
In this paper, combined rational and power functions are used to represent magnetization curves of high magnetic permeability ferromagnetic materials. The proposed functions cover much wider range of magnetic fields than functions currently used in simulation software packages. The objective is to present simple functions for approximation of magnetization curves with minimum number of unknown constants. The calculated functions are finally compared with measured magnetization curves to validate the precision in a wide field range from $10^{-2}$ to $10^{6} \mathrm{~A} / \mathrm{m}$.
\end{abstract}

Index Terms - Analytical functions, high magnetic permeability materials, curve fitting, magnetization curves .

\section{INTRODUCTION}

$\mathrm{T}$ HE most industrial used ferromagnetic materials could be categorized to two major groups of metallic and nonmetallic magnetic materials. Non-metallic magnetic materials are composed of Ferrite families. Metallic magnetic materials are mostly iron alloys families such as castiron and steels, low carbon steels, silicon steels, nickel based iron alloy and cobalt based iron alloy [1].

The nickel based iron alloys (Ni-Fe) have special industrial applications because of very high maximum permeability with small hysteresis losses and considerably high electrical resistivity. The high magnetic permeability is caused by small hysteresis loop and very low magnetic coercive force, which makes sharp changing of magnetic flux density at low magnetic field strength. Thesecharacteris tics shows suitable applications of Ni-Fe alloy for telecommunications functions . They have also numerous industrial applications such as magnetic sensors, high efficiency transformers, magnetic recording heads and magnetic shields [1] -[3].

Analytical representations of the magnetization and $B-H$ curve of magnetic materials are used for magnetic modeling, numerical analysis and design process. Using approximated mathematical $B$ - $H$ curve could help for first design step of magnetic devices [3]. It gives apparent and fast picture of maximum magnetic relative permeability and magnetic saturation without $B-H$ data table. $B-H$ curve could be represented by different closed-form formula [4]. Several publications have presented detailed analys is for $B-H$ curve modeling, for example, rational function [4] - [7] and exponential function [8] - [10]. The modeled magnetic materials for $B$ - $H$ functions were silicon steel laminations and solid irons and steels in [4] - [10], which have small magnetic permeability es pecially at low magnetic fields. Papers [5] and [7] are mostly devoted to optimization of curve fitting. Piecewise modeling of $B-H$ curve with high precision is also presented [11] but it does not obtain one closed-formequation. Power functions could precisely model a fraction part of $B-H$ curve but not the whole $B-H$ curve from low field part to highly saturated part [12]-[14].

Corresponding author: M. Mirzaei (e-mail: mirzameh@fel.cvut.cz).
In this paper rational function and power function are combined for modeling of very high permeability $B-H$ curves. The constants of the proposed function are calculated by curve fitting tool. It is shown that the proposed function can accurately fit the measured $B-H$ curve despite its notcomplicated equation. Finally, the proposed function is used for curve fitting of modified $B-H$ curve corresponding to fundamental component of flux density for AC analysis.

\section{BASIC STUDY}

\section{A. Assumptions}

Magnetization parameter, $J$ versus magnetic field strength and relative magnetic permeabilities are represented as following:

$J=B-\mu_{0} \cdot H$

$\mu_{r, a}=\frac{1}{\mu_{0}} \cdot \frac{B}{H}$

$\mu_{r, d}=\frac{1}{\mu_{0}} \cdot \frac{d B}{d H}=\frac{1}{\mu_{0}} \cdot \frac{d\left(\mu_{0} \cdot \mu_{r, a} \cdot H\right)}{d H}=\mu_{r, a}+H \cdot \frac{d \mu_{r, a}}{d H}$

where, $\mu_{0}, \mu_{r, a}$ and $\mu_{r, d}$ are free space magnetic permeability, apparent relative permeability and differential relative permeability. In order to calculate analytical function, it must be considered that magnetization, $J$ is becoming constant when magnetic field strength, $H$ is moving toward infinite and apparent and differential relative permeabilities must have one maxima between magnetic field strength, $H=0$ until $H=\infty$. The former condition is necessary to model Rayleigh region of the $B-H$ curve.

\section{B. Basicfunction}

First order rational function is simple analytical function, which could match with $B-H$ curve from low field to highly saturation. The main disadvantage of first order rational function is that it is not able to model Rayleigh region and relative permeability maxima could not be reproduced. In order to improve it, power of parameter, $x$ must be adjusted to value above 1 : 


$$
f(x)=\frac{a \cdot x^{b}}{1+a^{\prime} \cdot x^{b}},(f(x)=J, x=H)
$$

where, $a, a^{\prime}$ and $b$ are constants. The relative magnetic permeability for basic function (2) is as following:

$$
\begin{aligned}
& f(x)=\frac{a \cdot x^{b}}{1+a^{\prime} \cdot x^{b}},(J=f(x), x=H) \\
& \mu_{r, a}=1+\frac{1}{\mu_{0}} \cdot \frac{a \cdot x^{b-1}}{1+a^{\prime} \cdot x^{b}} \\
& \mu_{r, d}=\mu_{r, a}+\frac{1}{\mu_{0}} \cdot \frac{(b-1)-a^{\prime} \cdot x^{b}}{\left(1+a^{\prime} \cdot x^{b}\right)^{2}} \cdot a \cdot x^{b-1} \\
& =1+\frac{1}{\mu_{0}} \cdot \frac{a \cdot b \cdot x^{b-1}}{\left(1+a^{\prime} \cdot x^{b}\right)^{2}}
\end{aligned}
$$

Maximum values for relative magnetic permeability is given in (4). It is shown that smaller non-integer $b$ less than 2 makes it possible increase maximum relative magnetic permeability and decrease corresponding field strength, $H$ without changing parameter $a$ and $a^{\prime}$, which is required for high permeability magnetic materials modeling. Parameters $a$ and $a^{\prime}$ could be adjusted for matching formula for the modeling of the saturation and knee of $B-H$ curve. Fig. 1 shows schematic view of $B-H$ curves and relative magnetic permeabilities vers us magnetic field strength, which maximum value of differential relative permeability is always bigger than maximum value of apparent relative permeability.

$$
\begin{aligned}
& \mu_{r, a}\left(x=\sqrt[b]{\frac{b-1}{a^{\prime}}}\right)=\mu_{r, a}^{\max }=1+\frac{1}{\mu_{0}} \frac{a\left(\frac{b-1}{a^{\prime}}\right)^{\frac{b-1}{b}}}{b} \\
& \mu_{r, d}\left(x=\sqrt[b]{\frac{b-1}{a^{\prime} \cdot(b+1)}}\right)=\mu_{r, d}^{\max }=1+\frac{1}{\mu_{0}} \frac{a\left(\frac{b-1}{a^{\prime}(b+1)}\right)^{\frac{b-1}{b}}}{\frac{4 \cdot b}{(b+1)^{2}}}
\end{aligned}
$$

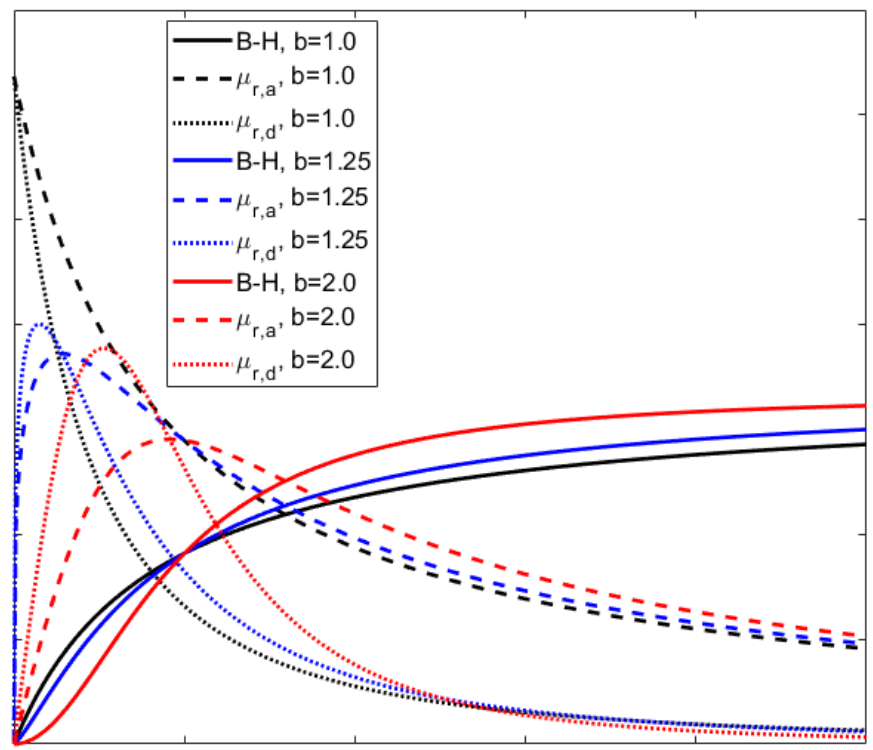

Fig. 1. Schematic $B$ - $H$ curve based on (2) - parameters $a$ and $a^{\prime}$ are the same for all curves

\section{PROPOSED FunCtion}

One possible formof the proposed function for combined rational function and power function could be as following:

$f(x)=\frac{\sum_{i=1,2, \ldots} a_{i} \cdot x^{b_{i}}}{1+\sum_{i=1,2, \ldots} a_{i}^{\prime} \cdot x^{b_{i}}},\left(f(x)=J^{m}, x=H^{n}\right)$

where, $a_{i}, a_{i}^{\prime}, b_{i}, m$ and $n$ are constants.

The big range of magnetic field streng th between the small values for maximum relative permeability and large values for high saturation make to choose $m$ and $n$ values not equal to one. The parameters $m$ and $n$ are adjusted to improve curve fitting process; they have positive values less than 1 . The relative apparent permeability and relative differential permeability are analytically calculated:

$$
\begin{aligned}
& \mu_{r, a}=1+\frac{1}{\mu_{0}} \cdot\left(\frac{\sum_{i=1,2, \ldots} a_{i} \cdot H^{n \cdot b_{i}-m}}{1+\sum_{i=1,2, \ldots} a_{i}^{\prime} \cdot H^{n \cdot b_{i}}}\right)^{\frac{1}{m}} \\
& \mu_{r, d}=1+\frac{1}{\mu_{0}} \cdot \frac{1}{m} \cdot\left(\frac{\sum_{i=1,2, \ldots} a_{i} \cdot H^{n \cdot b_{i}}}{1+\sum_{i=1,2, \ldots} a_{i}^{\prime} \cdot H^{n \cdot b_{i}}}\right)^{\frac{1-m}{m}} \times \\
& \frac{C_{1}-C_{2}}{\left(1+\sum_{i=1,2, \ldots} a_{i}^{\prime} \cdot H^{n \cdot b_{i}}\right)^{2}} \\
& C_{1}=\sum_{i=1,2, \ldots} a_{i} \cdot n \cdot b_{i} \cdot H^{n \cdot b_{i}-1} \cdot\left(1+\sum_{i=1,2, \ldots} a_{i}^{\prime} \cdot H^{n \cdot b_{i}}\right) \\
& C_{2}=\sum_{i=1,2, \ldots} a_{i} \cdot H^{n \cdot b_{i}} \cdot \sum_{i=1,2, \ldots} a_{i}^{\prime} \cdot n \cdot b_{i} \cdot H^{n \cdot b_{i}-1}
\end{aligned}
$$

Different high permeability materials are used from two material data resources [15]-[17] to validate the analytical functions.

\section{A. First Group ofMaterials [15], [16]}

In this part, three Ni-Fe magnetic materials are used for curve fitting. The number of power functions are limited to maximum three in the numerator and denominator of rational function:

$$
f(x)=\frac{a_{1} \cdot x^{b_{1}}+a_{2} \cdot x^{b_{2}}+a_{3} \cdot x^{b_{3}}}{1+a_{1}^{\prime} \cdot x^{b_{1}}+a_{2}^{\prime} \cdot x^{b_{2}}+a_{3}^{\prime} \cdot x^{b_{3}}}
$$

The number of unknowns in (7) are 9 with another unknown parameter $m$ and $n$, which will be totally 11 . The curve fitting process is used to find unknowns [18]. The parameters $m$ and $n$ are adjusted by trial and errors and they are not calculated by curve fitting to decrease the complexity of curve fitting. 


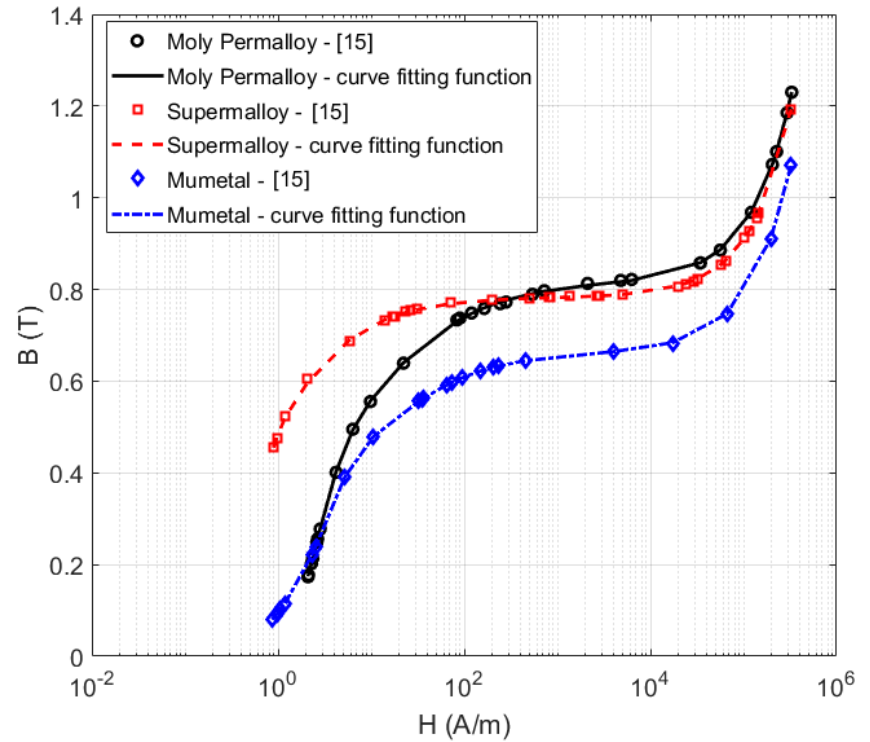

Fig. 2. The curve fitting for $B-H$ curve

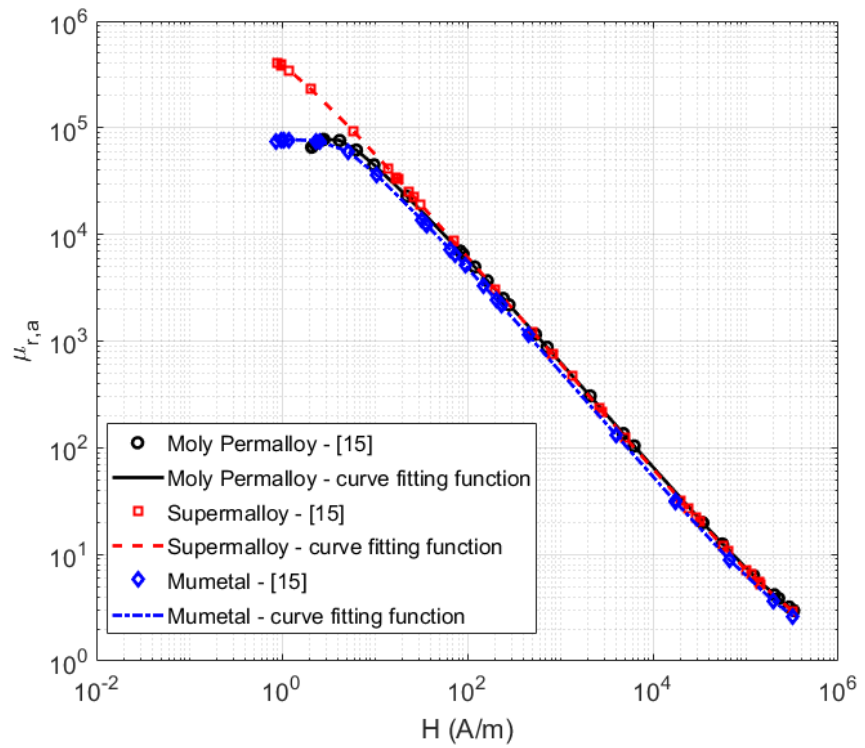

Fig. 3. The curve fitting for $\mu_{r, a}-H$ curve - apparent relative permeability

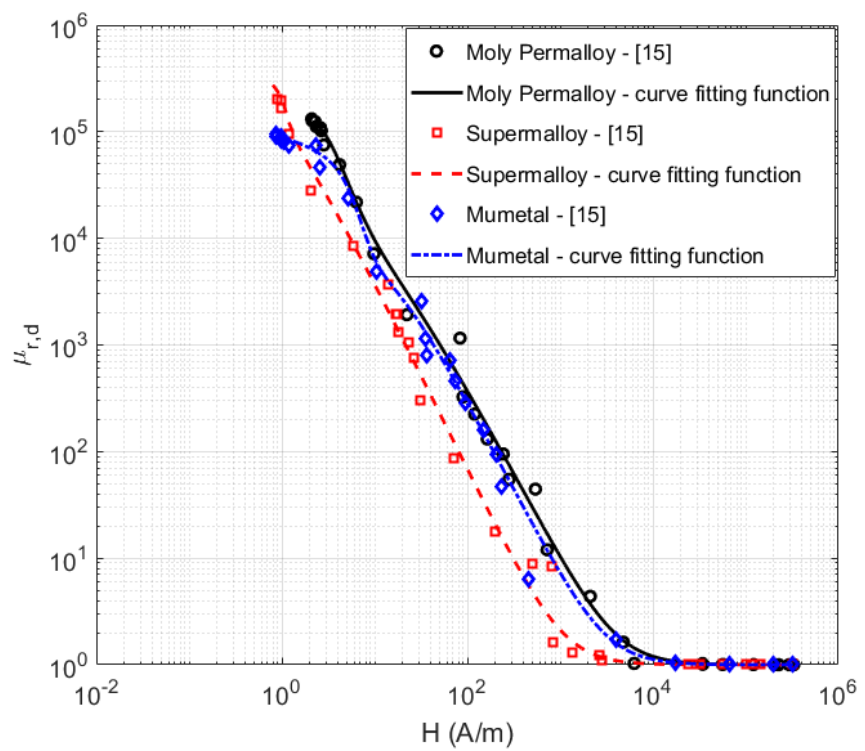

Fig. 4. The curve fitting for $\mu_{r, d}-H$ curve - differential relative permeability
The results are presented in Fig. 2 - Fig. 4 for three high permeability magnetic materials with relative permeability between 50000 and 500 000. The unknowns are calculated with curve fitting:

Moly Permalloy, $\quad a_{1}=-2.679, \quad a_{1}^{\prime}=-0.8315, \quad a_{2}=2.758$, $a_{2}^{\prime}=0.9487, \quad a_{3}=0.08104, \quad a_{3}^{\prime}=0.09077, \quad b_{1}=28, \quad b_{2}=27.74$, $b_{3}=39.48, m=0.56, n=0.07$

Supermalloy, $\quad a_{1}=17.37, \quad a_{1}^{\prime}=19.92, \quad a_{2}=0.52, \quad a_{2}^{\prime}=0.2364$, $a_{3}=0.8789, a_{3}^{\prime}=6.982, b_{1}=85.01, b_{2}=11.73, b_{3}=73.74, m=0.56$, $n=0.07$

Mumetal, $a_{1}=0.04578, a_{1}^{\prime}=0.0576, a_{2}=0.617, a_{2}^{\prime}=0.469, a_{3}=-$ $0.3419, a_{3}^{\prime}=-0.3305, b_{1}=33.42, b_{2}=15.65, b_{3}=22.79, m=0.56$, $n=0.07$

\section{B. Second Group of Materials [17]}

Two Ni-Fe magnetic materials are used for curve fitting from material data resource [17]. The calculated curves and analyticalfunctions unknown are presented in Fig. 5 -Fig. 7:

Metglas, $a_{1}=219.2, a_{1}^{\prime}=6661, a_{2}=722.9, a_{2}^{\prime}=770.4, a_{3}=0.2055$, $a_{3}^{\prime}=0.1705, b_{1}=5$.941, $b_{2}=7.817, b_{3}=11.3, m=0.56, n=0.63$

Nickel-Steel, $\quad a_{1}=0.05361, \quad a_{1}{ }_{1}=0.04131, \quad a_{2}=0.1532$, $a_{2}^{\prime}=0.09216, a_{3}=-0.04524, a_{3}^{\prime}=-0.02018, b_{1}=3.961, b_{2}=1.069$, $b_{3}=3.552, m=0.56, n=0.63$

Table I presents maximum and rms errors for five analyzed materials. The large value for maximum errors are due to the mis matching between curve fitting function and measured data at one point.

TABLE I

NORMALIZED RMS ERROR AND NORMALIZED MAXIMUM ERROR

\begin{tabular}{||l|c|c|c|c||}
\hline \multirow{2}{*}{ Material } & \multicolumn{2}{|c|}{ Normalized rms error (\%) } & \multicolumn{2}{c|}{ Max error (\%) } \\
\cline { 2 - 5 } & $B$ & $\mu_{\mathrm{r}}$ & $B$ & $\mu_{\mathrm{r}}$ \\
\hline Moly permalloy & 0.27 & 0.55 & 0.60 & 0.60 \\
\hline Supermalloy & 0.28 & 1.55 & 1.53 & 1.53 \\
\hline Mumetal & 0.20 & 0.64 & 0.90 & 0.90 \\
\hline Metglas & 0.45 & 0.87 & 2.1 & 2.1 \\
\hline Nickel-Steel & 0.29 & 1.20 & 13.21 & 13.21 \\
\hline
\end{tabular}

\section{Alternative Functions}

Shorter form of the proposed analytical function and standard rational function, (8) and (9) are also evaluated. Table II shows corresponding errors for using (8), which are totally increased in comparison with longer form function in (7).

The rational function with 5th order and 10 unknowns results are presented in Fig. 8. Two discontinuities are observed due to the zeros in denominator of rational function, which is common for rational function curve fitting. It needs complicated algorithm to remove zeros [5] and [7]. This phenomena has less probability to happen in the proposed analytical function because powers of parameter, $x$ is not an integer number as in (9). 


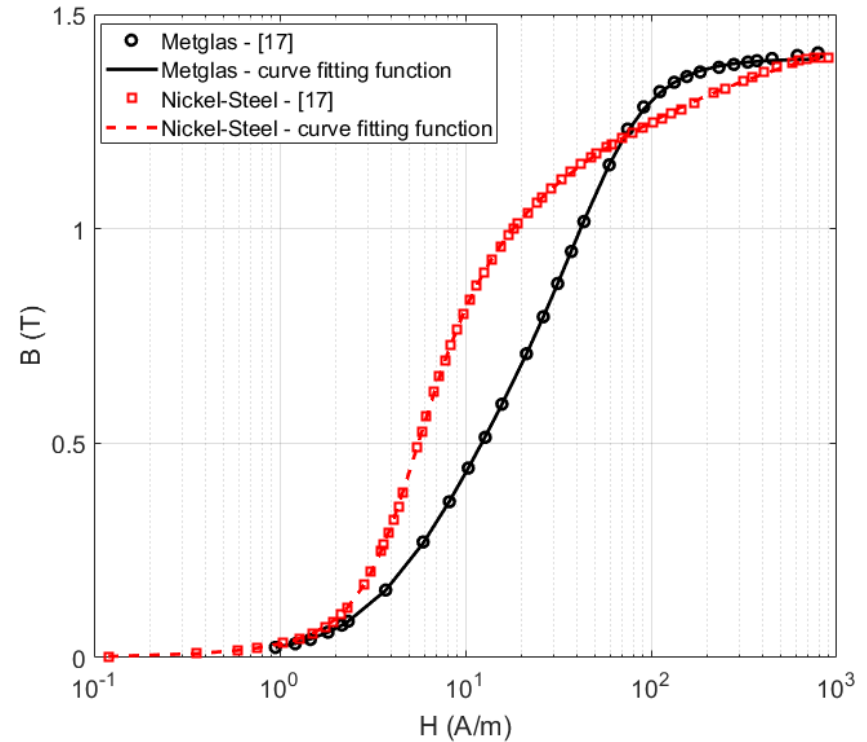

Fig. 5. The curve fitting for $B-H$ curve

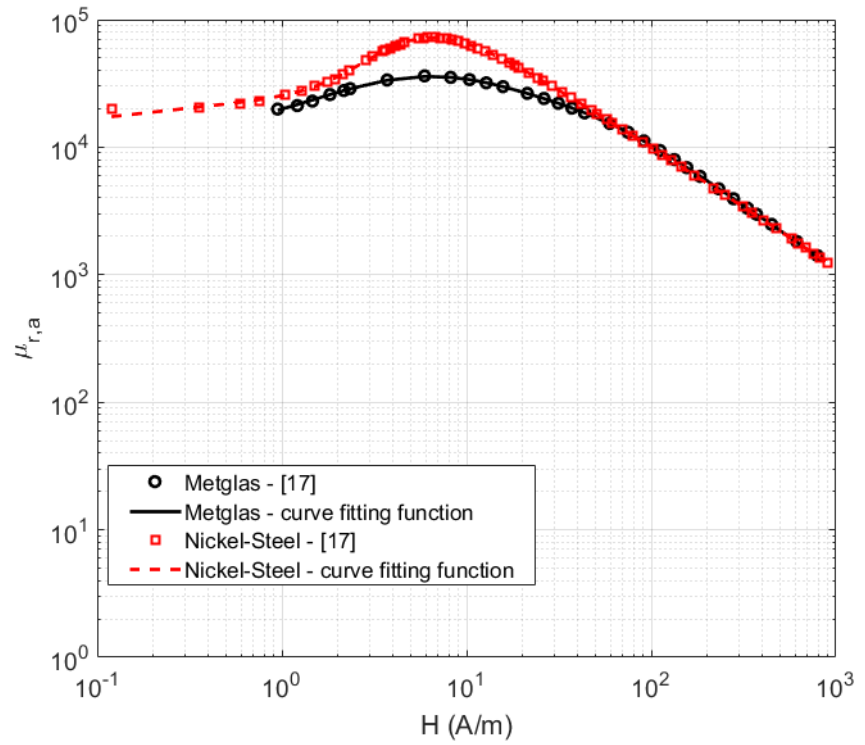

Fig. 6. The curve fitting for $\mu_{r, a}-H$ curve - apparent relative permeability

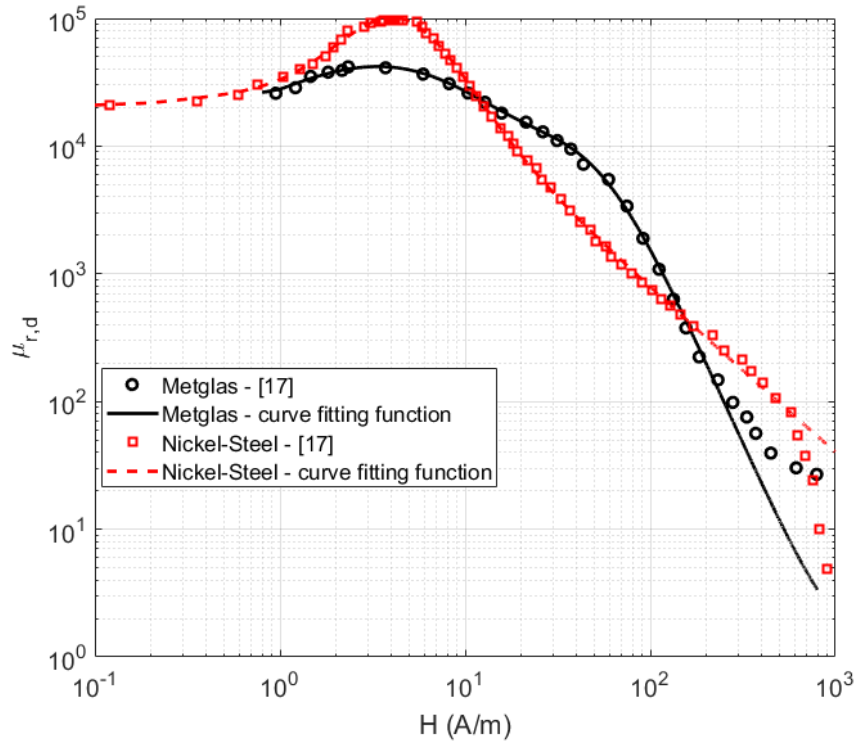

Fig. 7. The curve fitting for $\mu_{r, d}-H$ curve - differential relative permeability

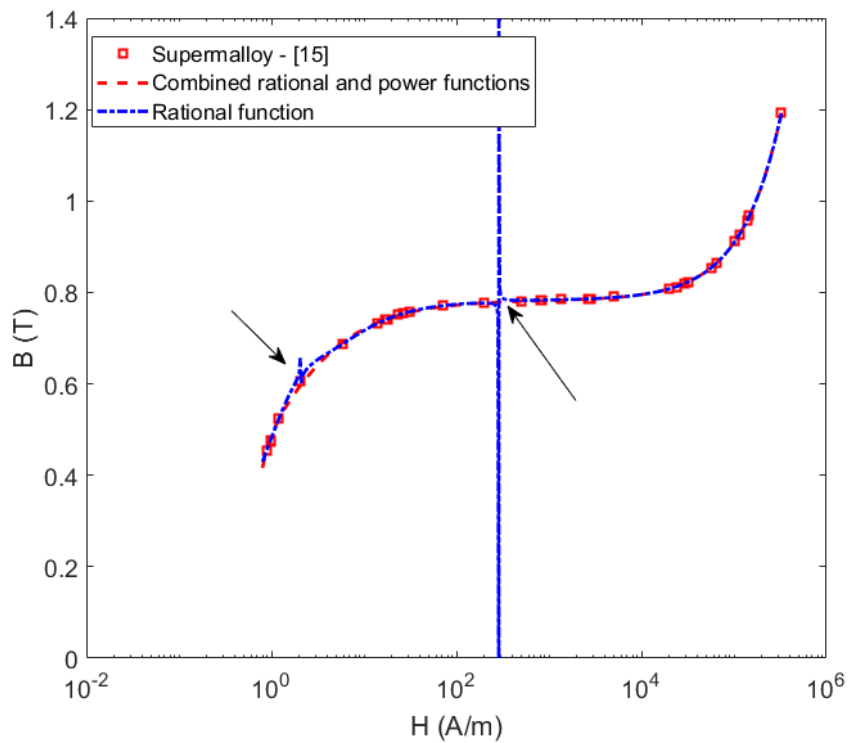

Fig. 8. The curve fitting for $B-H$ curve - calculated rational function, (9) unknowns: $a_{1}=0.7828, a_{2}=-228.1, a_{3}=969.1, a_{4}=-2581, a_{5}=3194, a_{1}^{\prime}=-290.6$, $a_{2}^{\prime}=937.9, a_{3}^{\prime}=-1792, a_{4}^{\prime}=634, a_{5}^{\prime}=3323$

$$
\begin{aligned}
& f(x)=\frac{a_{1} \cdot x^{b_{1}}+a_{2} \cdot x^{b_{2}}}{1+a_{1}^{\prime} \cdot x^{b_{1}}+a_{2}^{\prime} \cdot x^{b_{2}}},\left(f(x)=J^{m}, x=H^{n}\right) \\
& f(x)=\frac{a_{1} \cdot x+a_{2} \cdot x^{2}+a_{3} \cdot x^{3}+a_{4} \cdot x^{4}+a_{5} \cdot x^{5}}{1+a_{1}^{\prime} \cdot x+a_{2}^{\prime} \cdot x^{2}+a_{3}^{\prime} \cdot x^{3}+a_{4}^{\prime} \cdot x^{4}+a_{5}^{\prime} \cdot x^{5}} \\
& (f(x)=J, x=H)
\end{aligned}
$$

TABLE II

NORMALIZED RMS ERROR AND NORMALIZED MAXIMUM ERROR - FUNCTION WITH LESS UNKNOWNS

\begin{tabular}{||l|c|c|c|c||}
\hline \multirow{2}{*}{ Material } & \multicolumn{2}{|c|}{ Normalized rms error (\%) } & \multicolumn{2}{c|}{ Max error (\%) } \\
\cline { 2 - 5 } & $B$ & $\mu_{\mathrm{r}}$ & $B$ & $\mu_{\mathrm{r}}$ \\
\hline Moly permalloy & 0.59 & 2.06 & 2.62 & 2.62 \\
\hline Supermalloy & 0.26 & 0.684 & 1.32 & 1.32 \\
\hline Mumetal & 1.26 & 3.15 & 6.63 & 6.63 \\
\hline Metglas & 3.32 & 6.56 & 8.6 & 8.6 \\
\hline Nickel-Steel & 1.0 & 15.35 & 98.9 & 98.9 \\
\hline
\end{tabular}

The unknowns are calculated for (8) with curve fitting:

Moly Permalloy, $a_{1}=4.256, a_{1}^{\prime}=4.894, a_{2}=-4.337, a_{2}^{\prime}=-5.592$, $b_{1}=2.527, b_{2}=1.222, m=0.56, n=0.07$

Supermalloy, $a_{1}=0.6312, a_{1}^{\prime}=0.7258, a_{2}=-0.4198, a_{2}^{\prime}=-1.408$, $b_{1}=4.737, b_{2}=0.1899, m=0.56, n=0.07$

Mumetal, $a_{1}=0.4232, a_{1}^{\prime}=0.5815, a_{2}=0.003329, a_{2}^{\prime}=0.004199$, $b_{1}=15.23, b_{2}=30.72, m=0.56, n=0.07$

Metglas, $a_{1}=0.000468, a_{1}^{\prime}=0.0004867, a_{2}=-0.0002592, a_{2}^{\prime}=-$ $0.9988, b_{1}=0.6358, b_{2}=0.000674, m=0.56, n=0.63$

Nickel-Steel, $a_{1}=0.1039, \quad a_{1}^{\prime}=0.09879, \quad a_{2}=0.001024, \quad a_{2}^{\prime}=$ $0.0008324, b_{1}=2.629, b_{2}=4.219, m=0.56, n=0.63$

Other functions comparison such as exponential functions or closed-form trigonometric functions are skipped to be presented in this paper, which could give less precision rather than rational function. 


\section{Modified $B-H$ CuRve}

A modified $B-H$ curve is used when magnetic materials are involved in AC ortime harmonic analysis especially with high nonlinearity. The $B-H$ curve is modified to $B_{1}-H$, which $B_{1}$ is fundamental component of flux density when field strength changes in sinusoidal form [14] and [15].

Fig. 9 and Fig. 10 show $B_{1}-H$ curve from measured values and curve fitting function. The results are promising for $B_{1}-H$ curve. The calculated results for maximum error of flux dens ity and relative permeability, rms error of flux density and relative permeability are, $1.41 \%$ and $1.41 \%, 0.29 \%$ and $0.70 \%$, respectively.

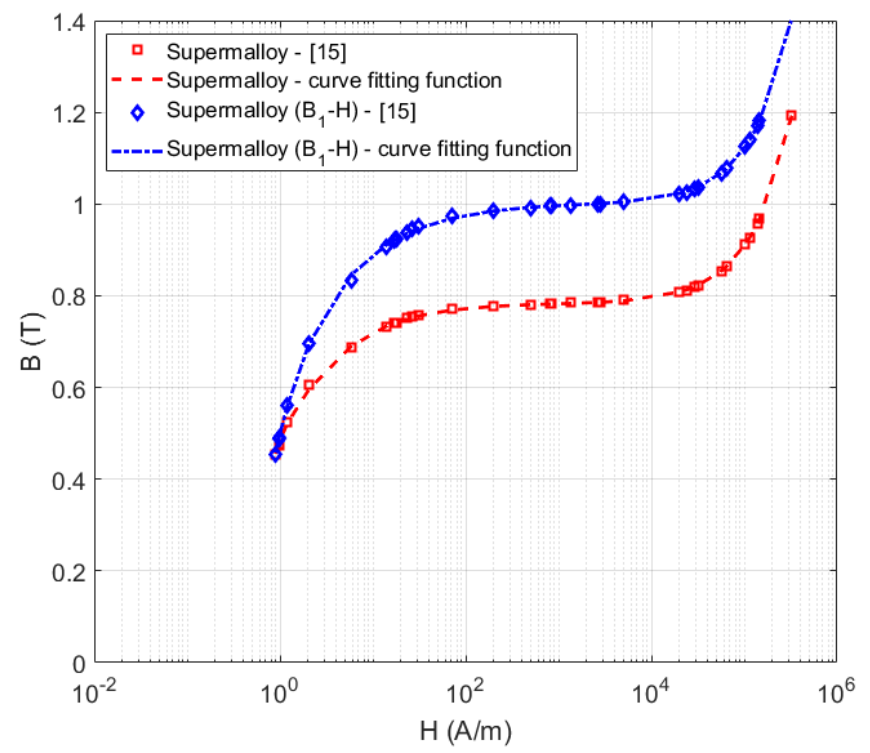

Fig. 9. The curve fitting for $B_{1}-H$ curve

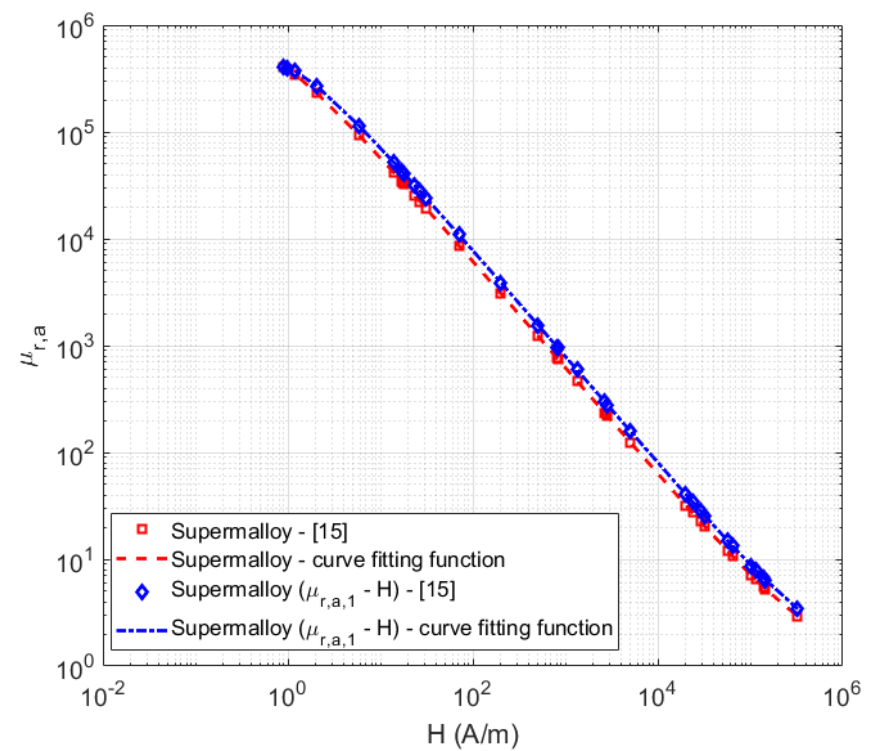

Fig. 10. The curve fitting for $\mu_{r, a, 1}-H$ curve - apparent relative permeability

\section{CONCLUSION}

New analytical function has been presented, which could precisely model $B-H$ curve and relative permeability. The selected materials were high magnetic permeability $\mathrm{Ni}-\mathrm{Fe}$ alloys but the presented analy tical function could alsobe used for other magnetic materials. The main advantages of presented function are its compact format and high precision even with low number of unknowns for curve fitting. The typical value of rms error ranges from 0.3 to $1.6 \%$.

Standard rational function was compared with the proposed analytical function, which has disadvantage of probable zeros in denominator and discontinuities in the modeled curve. Exponential functions for $B-H$ curve modeling are not as precise as rational functions. The unknowns of the proposed analytical function could be calculated with simple curve fitting function.

Compatibility of the proposed analytical function has been presented for modified $B-H$ curve corresponding to fundamental component of flux density, which shows also high precision.

\section{REFERENCES}

[1] C.W. Chen, Magnetism And Metallurgy Of Soft Magnetic Materials, North Holland , 1st January 1977

[2] P. Ripka, Magnetic Sensors and Magnetometers, Artech House, Jan 1, 2001 - Technology \& Engineering - 494 pages

[3] S. Tumanski, Handbook of Magnetic Measurements, June 23, 2011 by CRC Press Reference - 404 Pages

[4] F. C. Trutt, E. A. Erdélyi, and R. E. Hopkins, "Representation of the magnetization characteristic of dc machines for computer use," IEEE Trans. Power App. Syst., vol. 87, no. 3, pp. 665-669, Mar. 1968.

[5] G. F. T. Widger, "Representation of magnetisation curves over extensive range by rational-fraction approximations," Proc. Inst. Elect. Eng., vol. 116, no. 1, pp. 156-160, Jan. 1969.

[6] J. Rivas, J. M. Zamarro, E. Martín, and C. Pereira, "Simple approximation for magnetization curves and hysteresis loops," IEEE Trans. Magn., vol. 17, no. 4, pp. 1498-1502, Jul. 1981.

[7] Patrick Diez andJ. P. Webb,"A Rational Approach to B - H Curve Representation", IEEE Trans. on Magn. , Year: 2016, Volume: 52, Issue: 3

[8] M. K. El-Sherbiny, "Representation of the magnetization characteristic by a sum of exponentials", IEEE Trans. Magn., vol. MAG-9, no. 1, pp. 60-61, Mar. 1973.

[9] W. K. Macfadyen, R. R. S. Simpson, R. D. Slater, W. S. Wood, "Representation of magnetization curves by exponential series", Proc. IEE, vol. 120 , no. 8, pp. 902-904, 1973.

[10] J. R. Brauer, "Simple equations for the magnetization and reluctivity curves of steel," IEEE Trans. Magn., vol. 11, no. 1, p. 81, Jan. 1975.

[11] P. Diez, "Symmetric Invertible B - H Curves Using Piecewise Linear Rationals", IEEE Trans. on Magn. , Year: 2017, Volume: 53, Issue: 6

[12] Nejman, L.R., Skin effect in ferromagnetic bodies, GEI, MoscowLeningrad, 1949

[13] J Lammeraner, and M Stafl, Eddy Currents, Published by Iliffe, 1967

[14] S. A. Nasar; G. Y. Xiong; Z. X. Fu, "Eddy-current losses in a tubular linear induction motor", IEEE Trans. on Magn., 1994, Volume: 30, Issue: 4, Pages: 1437 - 1445,

[15] http://www.femm.info/wiki/Documentation/

[16] Metals Handbook, Volume 1, American Society for Metals, 1966

[17] Free BH Curves, accessed on March 02, 2018. [Online]. Available: http://www.magweb.us/free-bh-curves/

[18] https://www.mathworks.com/products/curvefitting.html 